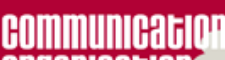
Oryanllatín.

\section{Communication et organisation}

Revue scientifique francophone en Communication organisationnelle

26 | 2005

La communication des nouvelles éthiques de l'entreprise

\title{
Espace public médiatisé et coopération transfrontalière
}

Le cas de la baie de Txingudi au Pays basque

Sergio Argul, Zoé Bray et Jean-Baptiste Harguindéguy

\section{CpenEdition}

\section{Journals}

Édition électronique

URL : https://journals.openedition.org/communicationorganisation/3293

DOI : 10.4000/communicationorganisation.3293

ISSN : 1775-3546

Éditeur

Presses universitaires de Bordeaux

Édition imprimée

Date de publication : 2 janvier 2005

Pagination : 223-234

ISSN : 1168-5549

\section{Référence électronique}

Sergio Argul, Zoé Bray et Jean-Baptiste Harguindéguy, «Espace public médiatisé et coopération transfrontalière », Communication et organisation [En ligne], 26 | 2005, mis en ligne le 19 juin 2012, consulté le 07 décembre 2022. URL : http://journals.openedition.org/communicationorganisation/ 3293 ; DOI : https://doi.org/10.4000/communicationorganisation.3293

Ce document a été généré automatiquement le 29 septembre 2020.

Tous droits réservés 


\section{Espace public médiatisé et coopération transfrontalière}

Le cas de la baie de Txingudi au Pays basque

Sergio Argul, Zoé Bray et Jean-Baptiste Harguindéguy

1 Les études sur le rapport entre le territoire et les médias se multiplient depuis quelques années. Nous souhaitons prolonger ces analyses en nous interrogeant sur l'existence d'un espace public médiatisé transfrontalier au Pays basque. La notion d'espace public médiatisé a été définie par D. Wolton ${ }^{1}$ à partir des travaux de J. Habermas ${ }^{2}$ qui a mis en évidence la constitution d'une sphère de communication indépendante depuis la Renaissance. Cependant, plutôt que d'envisager la médiatisation de cet espace comme une perversion, D. Wolton a montré que les démocraties contemporaines étaient consubstantiellement liées aux médias et qu'en ce sens l'espace public était plutôt un espace médiatisé. Nous tâchons d'analyser l'émergence d'un tel espace au sein d'un territoire frontalier. En effet, les accords de coopération transfrontalière se multiplient en Europe depuis vingt-cinq ans. Ces rapprochements politico-administratifs bouleversent les territoires traditionnels et favorisent l'émergence de nouveaux territoires fonctionnels transfrontaliers. Le cas d'étude retenu est la zone urbaine côtière regroupant les villes d'Hendaye (France), Irun et Fontarabie (Espagne), regroupées autour de la baie de Txingudi et séparées par la rivière Bidassoa qui délimite la frontière francoespagnole au Pays basque. Depuis 1998 ces municipalités sont réunies au sein d'une institution de coopération intercommunale transfrontalière appelée Consorcio.

2 La position géopolitique de la baie de Txingudi est particulière. La frontière communale y est aussi une frontière entre le nord (Iparralde en basque) et le sud (Hegoalde) du Pays basque, entre les provinces traditionnelles basques du Labourd et du Guipuzcoa, entre le Département des Pyrénées-Atlantiques et la Députationdu Guipuzcoa, entre la Région Aquitaine et la Communauté Autonome d'Euskadi (CAE), entre la France et l'Espagne, et jusqu'en 1986 entre la Communauté Economique Européenne et le reste de l'Europe. Véritable bouillon de cultures la baie de Txingudi dispose de médias spécifiques relayant l'activité transfrontalière de ses habitants. Peut-on parler d'espace public médiatisé pour 
autant $?^{3}$ Les mass-media peuvent-ils favoriser l'émergence d'un village transfrontalier, version modeste du village planétaire de Mac Luhan ${ }^{4}$ ? Et si cet espace existe, quelle est son ampleur?

3 Notre hypothèse de départ tend vers le néo-fonctionnalisme et repose sur le constat du passage d'une logique territoriale à une logique de flux sous la pression du progrès technologique. Selon nous un espace public médiatisé transfrontalier peut émerger en Txingudi puisque toutes les conditions techniques y sont réunies. Nous basons notre étude sur quatre types de médias (presse, radio, télévision, Internet) que nous divisons selon leur portée stato-nationale, régionale, locale ou transfrontalière. Nous avons limité notre recherche à l'aspect qualitatif des émetteurs (nature du média, histoire, orientation politique, contenu sémiotique). Cette enquête a été effectuée à partir de recherches bibliographiques, d'entretiens non directifs avec les acteurs locaux et d'un travail d'observation participante entre 2000 et 2002. Nos résultats tiennent en quatre points relatifs à chacun des médias analysés.

\section{La presse transfrontalière face aux contradictions du nationalisme basque}

4 Nous limitons notre étude à la presse généraliste. En France, les principaux quotidiens Le Monde, Libération et Le Figaro prennent le territoire français comme référence. Le transfrontalier y est traité dans les pages internationales.

5 Au niveau régional le journal Sud-Ouest exerce un monopole sur la presse régionale en Aquitaine. Sud-Ouest propose des versions locales du journal pendant la semaine et une édition uniforme le dimanche. Rédigé en français, Sud-Ouest est financièrement lié aux quotidiens espagnols Correo Español (Bilbao) et Diario Vasco (Saint-Sébastien). Ces deux journaux appartiennent au groupe Correo, principal actionnaire de la presse locale en Espagne ainsi que du journal monarchiste conservateur $A B C$. Episodiquement, Sud-Ouest rend compte de l'actualité outre Pyrénées mais traite celle-ci dans les pages internationales.

6 A une échelle locale, est apparu en 1993 l'hebdomadaire La Semaine du Pays basque, devenu propriété du groupe Sud-Ouest. Centré sur l'actualité du Pays basque, les nouvelles d' Hegoalde y sont traitées dans les pages internationales. En 2001 a été créé un nouveau quotidien, Le Journal du Pays basque. Ce journal francophone relate de façon précise l'actualité d'outre Bidassoa dans la rubrique Pays basque même s'il concerne principalement la partie française. C'est également le cas de l'hebdomadaire nationaliste basque modéré Enbata. A noter qu'aucun de ces journaux ne sont distribués côté espagnol. A Hendaye le mensuel nationaliste Bil informe de l'agenda culturel d'Irun et de Fontarabie. Malgré tout Bil reste centré sur l'actualité hendayaise et est écrit en français.

7 En Espagne les journaux stato-nationaux les plus importantssont $E l$ País, El Mundo, ABC et La Vanguardia. Parmi eux, seuls El País et $A B C$ contiennent des pages spécifiques à la communauté autonome dans laquelle ils sont distribués. Les éditions régionales d'El Mundo n'existent qu'en Catalogne, à Madrid et dans les Baléares. Son édition Euskadi a été abandonnée il y a quelques années. Les principaux journaux de la CAE sont le Correo Español et le Diario Vasco. Ce dernier est également le premier quotidien du Guipuzcoa. Ses commentateurs politiques son désormais très prudents en raison des menaces terroristes qui pèsent sur le journal et sur ses annonceurs. Le Diario Vasco traite les nouvelles du Pays 
basque français (surtout la côte basque) dans une rubrique spécifique tenue par un correspondant français. Au début des années 1980 sont apparus Deia etEgin. En raison de leurs liens respectifs avec le Parti Nationaliste Basque (démocrate chrétien) et Batasuna (nationaliste basque de gauche aujourd'hui interdit en Espagne pour ses liens supposés avec l'ETA -Euskadi Ta Askatasuna, Pays-Basque et Liberté-) ces journaux traitent les nouvelles d'Iparralde comme des faits locaux afin de conserver la cohérence du discours nationaliste et minimisent le rôle de la frontière (Bray, 2004). Leurs articles restent toutefois moins élaborés que s'ils étaient produits par un journal du Pays basque nord ce qui provoque un décalage avec la ligne éditoriale pan-basque. Les opinions partisanes exprimées dans Egin ont d'ailleurs poussé les autorités espagnoles à interdire le journal, aussitôt réapparu sous le nom de Gara.

Plusieurs journaux ont une vocation transfrontalière. C'est notamment le cas d'Egunkaria. Jusqu'à sa fermeture en février 2003 en raison de ses liens hypothétiques avec l'ETA il s'agissait du seul quotidien à être explicitement transfrontalier. Lancé en 1990 sur souscription populaire Egunkaria était rédigé en basque et incluait environ $10 \%$ d'informations relatives aux provinces basques françaises. Le transfrontalier était traité dans les pages locales 5 . Depuis juin 2003, Egunkaria a été remplacé par le journal Berriak. En Txingudi, La Bahía de Txingudi et Bidaberri, journaux créés respectivement à Irun et dans la ville navarraise voisine de Bera de Bidasoa, sont rédigés en trois langues (français, espagnol, basque) et rapportent les informations relatives aux communes de l'embouchure de la Bidassoa. A Irunest aussi vendu El Irunes, un mensuel d'histoire et Irunero, produit par la municipalité afin de fomenter l'usage du basque. Enfin, le Consorcio propose sa propre revue Bidasoa-Txingudi. Edité à Irun, ce mensuel trilingue est distribué gratuitement. Sa particularité est de relativiser la frontière en publiant des publicités françaises et espagnoles, en insistant sur les points historiques rapprochant les deux rives et en adressant régulièrement des conseils aux habitants résidant dans un Etat différent de leur lieu de travail ou d'étude.

\section{Radios transfrontalières : vers un espace d'expression bascophone}

Nous limitons nos recherches aux stations de radio généralistes. Côté français, France Inter , RMC (Radio Monte Carlo), RTL (Radio Télévision Luxembourgeoise) et Europe1, pour ne citer que les plus importantes, peuvent être captées jusqu'à Hendaye. Ces radios émettent en général depuis Paris et prennent pour référence le territoire français. Leur influence est contrebalancée par Sud Radio, radio régionale installée à Toulouse et par un réseau dense de radios locales bascophones apparues après la libéralisation des ondes en 1981. En Iparralde, chaque province dispose de sa propre radio: Gure Irratia pour le Labourd, Irulegiko Irratia en Basse-Navarre, Xiberoko Botza en Soule. Irulegiko Irratia a été la première à établir des contacts réguliers avec ses homologues basques espagnols Xorroxin Irratia et Euskal Herri Irratia (Navarre) et Egin Irratia (Guipuzcoa). Enfin, France Bleu Pays Basque, antenne locale de Radio France installée à Bayonne, émet des programmes dans le cadre du Pays basque français. Elle maintient un correspondant à Saint-Sébastien qui intervient chaque matin pendant le bulletin d'information locale. Cette station joue pourtant plus le rôle de correspondant local pour France Inter que celui de radio locale. En Espagne, si jusqu'en 1977 seule la radio nationale RNE (Radio Nacional de España) pouvait donner des informations que les autres stations se contentaient de relayer, la situation a bien changé 
et de nombreuses radios sont apparues ${ }^{6}$. A Irun et Fontarabie, RNE1, RNE2, RNE3, Cadena SER, Onda Cero, Cadena COPE et Antena3 radio se partagent l'espace radiophonique. Ces radios sont pour la plupart basées à Madrid et couvrent les informations du territoire espagnol. Les radios régionales sont nombreuses et disposent de moyens financiers importants : Euskadi Irratia (bascophone) et Radio Euskadi (hispanophone) sont propriétés de la CAE, Xorroxin Irratia, Euskal Herri Irratia et Egin Irratia sont indépendantes. Parmi ces radios, seules Radio Euskadi et Euskadi Irratia donnent sporadiquement des nouvelles du Pays basque nord, d'autant plus qu'un accord lie Euskadi Irratia à Gure Irratia. A Irun, Radio Irun appartient au réseau SER et transmet les informations relatives à Irun et à Fontarabie. Radio Irun couvre l'actualité d'Hendaye de façon irrégulière. La seule radio pouvant être qualifiée de transfrontalière est Antxeta Irratia, antenne hendayaise de la radio bascophone Gure Irratia. L'influence de Gure Irratia à Hendaye était limitée par des problèmes de réception jusqu'à l'implantation d'un studio local en février 2002. Antxeta Irratia est spécifiquement tournée vers le public d'Hendaye, Irun et Fontarabie et emploie une petite équipe originaire des trois communes. Emettant exclusivement en basque, ses programmes restent inaccessibles à une large part de la population.

\section{La télévision transfrontalière entre volontarisme et manque de moyens}

10 Nous limitons notre analyse au réseau hertzien. Côté français, les chaînes émises depuis Paris dominent largement le temps d'antenne ${ }^{7}$. TF1, France 2 et France 3, Canal+, ARTE La Cinquième et $\mathrm{M} 6$ sont les seules à maintenir une programmation continue. Leur espace de référence est le territoire français. Même France 3 , envisagée dès sa création comme la chaîne des régions n'a jamais totalement rempli sa tâche. France 3 reste le seul média d'envergure à s'intéresser au local par le biais de décrochages locaux et régionaux. En outre, en plus des informations régionales d'Aquitaine retransmises depuis Bordeaux, la chaîne propose depuis 1992 un journal en basque et en français produit par son agence bayonnaise. Dans les années 1980 elle a également participé à la production de l'émission transfrontalière Pyrénées-Pirineos en collaboration avec les chaînes régionales espagnoles frontalières. En Espagne, les chaînes stato-nationales TVE 1 (Televisión Española), TVE2, Antena 3, Canal+ et Telecinco prennent en compte l'ensemble du territoire espagnol. Cependant, TVE 1, TVE 2 et Antena 3 maintiennent des rédactions spécifiques dans chaque communauté autonome et effectuent des décrochages régionaux et locaux durant les pauses publicitaires et certains journaux télévisés. La réelle spécificité de l'espace télévisuel espagnol réside dans le nombre élevé de chaînes régionales. Au sein de la CAE deux chaînes publiques dépendant du gouvernement autonome émettent en continu ${ }^{8}$ : ETB1 (Euskal Telebista - bascophone) et ETB2 (hispanophone). ETB1 maintient un correspondant permanent en France depuis le début des années 1980 et collabore avec les services locaux de France 3 Pays basque. En 1992 une rédaction a été établie à Bayonne pour produire un journal de sept minutes sur l'Iparralde. Après avoir longtemps retransmis ses programmes côté français en enfreignant la législation française sur la radiodiffusion, ETB1 a trouvé un accord avec le Comité Supérieur de l'Audiovisuel lui permettant depuis 1995 d'entretenir vingt-six émetteurs sur le sol français. A contrario, les Espagnols ne reçoivent pas la télévision française du fait du standard d'émission SECAM adopté par la France. 
11 Le seul média méritant a priori le qualificatif de transfrontalier est Localia Txingudi(ex : Txingudi Telebista), télévision locale créée à Irun en 1996 et qui couvre l'ensemble des trois villes de la baie. Cette vocation la rapproche idéologiquement du Consorcio sans toutefois la confondre institutionnellement avec ce dernier. Localia Txingudi possède son propre siège et emploie six personnes. En 2002 elle a intégré le réseau Localia du groupe Prisa, éditeur d'El País et de la Cadena SER. Le caractère transfrontalier de Localia Txingudi s'exprime à travers des programmes ayant trait au sport, aux enfants, aux personnalités, au patrimoine et à l'actualité de la baie. La chaîne produit plus de six heures d'émissions hebdomadaires. Pour le reste elle recourt aux informations du réseau Bloomberg et à des vidéos musicales. Malgré la volonté initiale de s'imposer en tant que média transfrontalier, les concepteurs de Localia Txingudi se sont trouvés dans l'obligation de recentrer leur activité sur la rive espagnole comme en attestent les titres de leurs programmes. D'une part des problèmes techniques de réception sont apparus à Hendaye, d'autre part la population hendayaise n'a jamais manifesté un grand intérêt pour la chaîne. En outre, peu d'entrepreneurs français achètent des espaces publicitaires. Très déçu, l'un des fondateurs de la chaîne estime même que «le transfrontalier, ici, ça n'existe pas ». La production d'émissions trilingues est une véritable gageure pour une entreprise aux moyens aussi limités ${ }^{9}$. Le personnel de Localia Txingudi est majoritairement hispanophone même si certains employés parlent un peu le basque et le français. Après une brève période (2001) durant laquelle, avec l'aide d'une journaliste temporaire francophone, Localia Txingudi a couvert les informations à Hendaye en français, la chaîne a renoncé à cette tâche, préférant se concentrer sur les actualités d'Irun et de Fontarabie.

\section{Le réseau Internet et la multiplication des frontières}

12 Autrefois considérées comme des cités pionnières les communes utilisant l'Internet sont aujourd'hui devenues majoritaires. Il n'existe toutefois qu'un seul site spécifiquement réservé à la baie de Txingudi : Bidasoa-Txingudi.com. Ce site a été développé par le Consorcio comme un outil d'information et de «conscientisation » citoyenne. A mi-chemin entre le modèle de la vitrine et celui du guichet ${ }^{10}$, ce site propose de découvrir les réalisations et les projets du Consorcio, l'agenda des manifestations de la baie et des adresses et numéros de téléphone utiles. Il est en outre possible d'envoyer ses questions et de télécharger des documents administratifs. Une liste de liens permet d'atteindre les sites des autres institutions de la baie comme Localia Txingudi ou d'accéder aux organisations transfrontalières externes comme l'Eurocité Basque Bayonne Saint-Sébastien. Un effort spécial a été apporté à la navigation qui peut être effectuée dans les trois langues de la baie. Ce site cherche à fédérer tous les habitants de la baie sans distinction et tâchent de refléter autant qu'influencer leur opinion. Néanmoins, le contenu du site n'est pas régulièrement renouvelé et les versions française et basque contiennent des erreurs de syntaxe, conséquences probables d'une traduction littérale à partir de l'espagnol. Ce site a d'ailleurs été conçu sous la responsabilité de Bidasoa Activa l'agence espagnole de développement travaillant pour Irun et Fontarabie ainsi que pour le Consorcio.

13 Bidasoa-Txingudi.com est concurrencé par d'autres sites, tournés vers un seul des côtés de la frontière. S'il existe une division claire entre Hendaye d'un côté et Irun et Fontarabie de l'autre, cette division n'a pas le même sens selon le site en question. Pour Hendaye.com, Irun.org et Hondarribia.com produits par les municipalités de la baie, il s'agit d'une division entre communes. Tout en proposant un contenu proche de celui de Bidasoa-txingudi.com 
ces sites délèguent les informations à caractère transfrontalier à ce dernier. Pour Txingudi.com qui fournit seulement des informations pratiques sur Irun et Fontarabie il s'agit également d'une division locale. Mais pour Bogaboga.com, Infobasque.com, Euskadi.net et Cybereuskadi.com, la frontière devient régionale. Les deux premiers sites sont spécialisés dans le traitement de la partie basque française alors que les deux autres se concentrent sur la partie basque espagnole.

On peut douter du caractère transfrontalier des sites Internet consacrés à la baie de Txingudi. Pour reprendre la distinction établie par M. Castells ${ }^{11}$ entre virtual community et network society, on peut affirmer que ces sites, tant par leur format que par leur contenu, favorisent l'apparition de communautés virtuelles thématiques articulées autour de chats, de forums et de mailing lists, mais que la création d'une société transfrontalière en réseau est encore une utopie ${ }^{12}$.

\section{La superposition d'espaces publics}

Nous sommes face à une situation contradictoire. Tous les moyens techniques de communication sont réunis pour qu'apparaisse un espace public médiatisé transfrontalier autour de la baie de Txingudi et pourtant celle-ci reste largement dominée par une logique stato-nationale côté basque français et régionale/stato-nationale côté basque espagnol.

Le complexe médiatique transfrontalier de la baie de Txingudi est fragile. Le contenu de ses messages est souvent contradictoire. La coopération n'est prônée clairement que par une poignée de médias. Qui plus est, les limites posées par les différentes sensibilités identitaires, la méconnaissance des langues, le cadre juridique et économique, semblent pour l'heure insurmontables.

Plutôt qu'un espace public médiatisé transfrontalier c'est plutôt la superposition de différents espaces publics qui semble caractériser la baie de Txingudi. Les frontières ne disparaissent pas, elles évoluent ${ }^{13}$ et continuent d'exercer une fonction de filtre cognitif ${ }^{14}$. En conclusion, nous nous rangeons volontiers aux côtés de D. Wolton ${ }^{15}$ lorsqu'il affirme que : "Les sociétés ouvertes doivent disposer d'un moyen de relation à l'autre : c'est la fonction de l'information, récit d'un monde qui élargit sans cesse ses frontières, mais qui s'adresse à une communauté bien particulière beaucoup plus réduite, et en général, nationale ».

\section{BIBLIOGRAPHIE}

Barth F., Ethnic Groups and Boundaries. The Social Organization of Cultural Differences. Boston, Brown, 1969.

Boure R., « Régions frontalières, télévision et communication électronique » in : Sciences de la Société. nº 37, 1996. p. 111-128.

Bray Z., Living Boundaries : Frontiers and Identity in the Basque Country. PIE -Peter Lang, Brussels, 2004. 
Bustamante E. et Zallo R., (sous la direction de) Las industrías culturales en España. Madrid, Akal, 1988.

Castells M., The Internet Galaxy. Reflections on the Internet, Business, and Society. Oxford, Oxford University Press, 2001.

Chalaby J.K., « Transnational Television in Europe. The Role of Pan-European Channels » in : European Journal of Communication. vol. 2, $\mathrm{n}^{\circ}$ 17, 2002. p. 183-203.

Darre A., « Médias et société en Pays basque. Vers la constitution d'un espace de la communication transfrontalier » in : Les Cahiers du LERASS - Recherches en Sciences de la Société. $\mathrm{n}$ - 25, 1992. p. 11-29.

Gunther R., Montero J.R. et Wert J.I., The Media and Politics in Spain, Barcelona, ICPS, 1999.

Habermas J., L'espace public : archéologie de la publicité comme dimension constitutive de la société bourgeoise, Paris, Payot, 1997.

Halavais A., « National Borders on the World Wide Web » in : New Media and Society. vol. 1, $\mathrm{n}^{\circ}$ 2, 1999. p. 12-28.

Kukawka P., « Politiques de communication et d'images des villes de l'Arc Atlantique » in : Revue Communication et Organisation. , $2^{\circ}$ semestre, $n^{\circ} 6,1994$. p. 23-37.

Palmer M. et Sorbets C., « France » in : Østergaard B.S. (sous la direction de) The Media in Western Europe. London, Sage, 1992. p. 57-74.

Raffestin C., « Eléments pour une problématique des régions frontalières » in : L'Espace géographique. Tome III, n 1, 1974. p. 110-132.

Ricaud P., « Les médias comme indicateurs, producteurs et acteurs d'espaces transfrontaliers. Développement de l'exemple basque et regards sur la situation catalane » in : Borja A., Letamendia F. et Sodupe K. (sous la direction de) La construcción del espacio vasco-aquitano. Bilbao, UPV-EHU, 1998. p. 289-307.

Vedel T., «L'Internet et les villes : trois approches de la citoyenneté » in : Hermès. n 26-27, 2000. p. $247-262$.

Wolton D., « Les contradictions de l'espace public médiatisé » in : Hermès. n 10, 1991. p. 95-114.

\section{NOTES}

1. Wolton Dominique, « Les contradictions de l'espace public médiatisé » in : Hermès. $\mathrm{n}^{\circ} 10,1991$. p. 95-114.

2. Habermas Jürgen, L'espace public: archéologie de la publicité comme dimension constitutive de la société bourgeoise. Paris, Payot., 1997.

3. Kukawka Pierre, «Politiques de communication et d'images des villes de l'Arc Atlantique » in : Revue Communication et Organisation. $2^{\circ}$ semestre, $\mathrm{n}^{\circ}$ 6, 1994. p. 23-37.

4. Boure Robert, «Régions frontalières, télévision et communication électronique ", in : Sciences de la Société. $\mathrm{n}^{\circ}$ 37, 1996. p. 111-128

Darre Alain (1992), «Médias et société en Pays basque. Vers la constitution d'un espace de la communication transfrontalier» in : Les Cahiers du LERASS - Recherches en Sciences de la Société. $\mathrm{n}$ - 25, 1992. p. 11-29.

5. Ricaud Pascal, «Les médias comme indicateurs, producteurs et acteurs d'espaces transfrontaliers. Développement de l'exemple basque et regards sur la situation catalane », in : 
Borja Anton, Letamendia Francisco et Sodupe Kepa (sous la direction de.), La construcción del espacio vasco-aquitano. Bilbao, UPV-EHU, 1998. p. 289-307.

6. Gunther Richard, Montero José Ramón et Wert José Ignacio, The Media and Politics in Spain. Barcelona, ICPS, 1999.

7. Palmer Michael et Sorbets Claude (1992), «France» in: Østergaard Bernt Stubbe (sous la direction de), The Media in Western Europe. London, Sage, 1992. p. 57-74.

8. Bustamante Enrique et Zallo Ramón, Las industrías culturales en España. Madrid, Akal, 1988.

9. Chalaby Jean K., "Transnational Television in Europe. The Role of Pan-European Channels » in : European Journal of Communication. vol. 2, $\mathrm{n}^{\circ}$ 17, 2002. p. 183-203.

10. Thierry Vedel distingue quatre possibilités d'utilisation d'Internet pour une commune: L'Internet- vitrine qui consiste à diffuser des informations vers le grand public et les employés municipaux avec une adresse électronique permettant de poser des questions; l'Intranet de gestion interne des services municipaux; l'Internet-guichet qui fonctionne comme un service d'informations municipales en ligne où l'on peut télécharger des documents, poser des questions et se renseigner sur les démarches administrative; l'Internet-forum qui permet aux habitants d'effectuer des propositions voire de voter sur des points particuliers. Vedel Thierry, «L'Internet et les villes : trois approches de la citoyenneté », in : Hermès. n²6-27, 2000. p. 247-262.

11. Castells Manuel, The Internet Galaxy. Reflections on the Internet, Business, and Society. Oxford, Oxford University Press, 2001.

12. Halavais Alexander, "National Borders on the World Wide Web ", in : New Media and Society. vol. 1, n 2, 1999. p. 12-28.

13. Barth Frederik, Ethnic Groups and Boundaries. The Social Organization of Cultural Differences, Boston, Brown, 1969.

Bray Zoé, Living Boundaries: Frontiers and Identity in the Basque Country. PIE -Peter Lang, Brussels, 2004.

14. Raffestin Claude, «Eléments pour une problématique des régions frontalières ", in : L'Espace géographique, tome III, $\mathrm{n}^{\circ} 1,1974$. p. 110-132.

15. Wolton Dominique, «Les contradictions de l'espace public médiatisé » in: Hermès. $n^{\circ} 10$, 1991. p. 95-114.

\section{RÉSUMÉS}

Dans cet article nous tâchons de vérifier l'existence d'un espace public médiatisé au sein de la zone frontalière constituée par les communes d'Hendaye (France), Irun et Fontarabie (Espagne) situées sur la côte Atlantique, autour de la baie de Txingudi au Pays basque. Depuis 1988 ces villes sont réunies au sein d'une institution de coopération transfrontalière intercommunale appelée Consorcio. Malgré l'existence de médias à vocation transfrontalière, la situation de la baie se caractérise plus par la superposition de différents espaces publics médiatisés locaux, régionaux et stato-nationaux que par l'émergence d'un véritable espace médiatique transfrontalier.

In this article, we examine the existence of a public media space in the frontier area of the towns of Hendaye (in France), and Irun and Hondarribia (in Spain), located on the Atlantic coast around the bay of Txingudi in the Basque Country. Since 1988, these three towns are linked together by a cross-frontier agreement called Consorcio Bidasoa-Txingudi. In spite of the potential existence of a cross-frontier space however, the situation in the bay area is characterised more by the 
overlapping of various local, regional and state-national public media spaces than by the emergence of any concrete cross-frontier one.

\section{AUTEURS}

SERGIO ARGUL

Diplômé en sciences de la communication à l'Université Complutense, S. Argul est membre de la section d'Etudes Internationales de l'Ecole Diplomatique Espagnole de Madrid. Il travaille actuellement sur le rôle des médias dans la transition démocratique.

\section{ZOÉ BRAY}

Anthropologue et docteur en sciences politiques et sociales à l'Institut Universitaire Européen de Florence (IUE), ses recherches portent sur l'impact des fonds structurels dans les régions frontalières.

\section{JEAN-BAPTISTE HARGUINDÉGUY}

Spécialiste de l'analyse des politiques publiques, J.B. Harguindéguy est doctorant à l'IUE où il travaille sur les politiques communautaires de coopération transfrontalière. 